\title{
Telecommunication Traffic Unit and Traffic Mathematical Model
}

\section{Sigit Haryadi}

April 2018

Citation: Haryadi, S. (2018, April 13). Telecommunication Traffic Unit and Traffic Mathematical Model. Retrieved from https://osf.io/jqxyd/. http://doi.org/10.17605/OSF.IO/JF5RY

\begin{abstract}
Here is described mathematical model of telecommunication traffic, that are about Traffic Unit, Theoretical Model of Telecommunication Traffic, Traffic Process, Traffic at Packet Level, Description of Point Processes, Characteristics of Point Process, Poisson Process, The concept of the Birth and Death Process, and Creating Mathematical Model of the Telecommunication Traffic.
\end{abstract}

\section{Traffic Unit}

Telecommunication traffic can be expressed in terms of the intensity of traffic and traffic volume. In detail, measurement of traffic intensity associated with planning and technical performance of the network, and measurement of the volume of traffic associated with the revenue of telecommunication Operators and Providers.

\subsection{Intensity of Traffic}

Definition of traffic intensity by ITU-T contained in ITU-T B.18:

The instantaneous traffic intensity in a pool of resources is the number of busy resources at a given instant of time (ITU-T B.18).

Note: Resource pool can be the number of trunks, number of channels, the number of timeslots and so forth depending on the technology used.

Because the resource pool on Unit Standard International is not having dimension (dimensionless), the unit of telecommunications traffic intensity also has no dimension. And the suggestion of many telecom experts who are members of the ITU-T Study Group unit of erlang is used (commonly abbreviated as erl. or E). Unit of telecommunications traffic intensity recommended by the ITU-T are also standardized by ISO. Using the unit of telecommunications traffic erlang for intensity began in 1946 as a tribute to the Danish mathematician AK Erlangen (1878 - 1929) who first put forward the theory of telephone traffic. 
Traffic unit of telecommunications traffic intensity on the telecommunications network based on IP (Internet Protocol) is still using Erlang. It should be noted that the Erlang which is dimensionless, suitable for use in modeling, telecommunications traffic, and can be used for all kinds of telecommunication services.

The intensity of the traffic used in telecommunication traffic modeling for planning purpose, telecommunication system, to control traffic and for traffic and performance monitoring of telecommunications services.

A simple explanation of the Erlang is as follows:

a) In a medium of transmission that is a bandwidth of $1 \mathrm{G}$ bps internet traffic flowing at $0.6 \mathrm{G}$ bps, the traffic intensity in the transmission medium is $=0.6 \mathrm{G}$ bps $/ 1 \mathrm{G}$ bps $=$ 0.6 Erlang.

b) On a web server that can serve 1000 hits per hour, there were 400 hits per hour, the traffic intensity on this web server is $=400$ hits per hour $/ 1000$ hits per hour $=0.4$ Erlang.

c) On a call center that is able to serve customers as many as 500 calls per minute there are 230 calls per minute, then the traffic intensity in the call center is a call per minute $=230$ calls per minute $/ 500$ calls per minute $=0.46$ Erlang.

\subsubsection{Erlang Problem and Solution Examples}

Calculate the intensity of traffic (using Erlang unit) on the 3 problems below:

a) A radio-link with a capacity of 6 channels, with measurements for 30 minutes, every channel average holded for 15 minutes.

b) A measurement of the digital switch over for 25 minutes, processed about 25,000 calls, which have a mean holding time $=3$ minutes $(=3$ minutes $/$ call $)$

c) A web server has a service rate $=1 \mathrm{M}$ bit per seconds, if in a 10-minute measurements received 600 requests, each request requires an average of 100 packet data @ 1,000 bits per packet.

Solution : final calculation should be dimensionless, because the requested unit is erlang !!

a) Traffic Intensity $=A=6$ channel $x$ ( 15 minutes per channel $/ 30$ minutes $)=3$ erlang.

b) Traffic Intensity $=\mathrm{A}=25,000$ calls $\mathrm{x} 3$ minutes per call $/ 25$ minutes $=3,000$ erlang.

c) Traffic Intensity $=A=(600$ requests $\times 100$ packets per request $x 1,000$ bits per packet $) /(1 \mathrm{M}$ bits per second $\mathrm{x}(10$ minutes $\mathrm{x} 60$ seconds per minute $)=0.1$ erlang 


\subsection{Traffic Volume}

The volume of traffic is a traffic that is served by telecommunication systems (= Carried traffic) over a period of time measurement.

A unit of telecommunications traffic volume depending on how revenues are calculated. The following gives examples of calculation and use of a unit of volume of traffic:

1. An Internet Service Provider determines that customers will have to pay $\$ 1$ per $1 \mathrm{G}$ byte of traffic used by customers, and then in this case, the unit volume of traffic is $\boldsymbol{G}$ byte. If in a month, the Internet Service Provider to get the volume of traffic at 1 million $\mathrm{G}$ byte, then the income $=1$ million $\mathrm{G}$ byte $\mathrm{x} \$ 1$ per $\mathrm{G}$ byte $=\$ 1$ million.

2. A Telephony Service Provider determines that customers will have to pay 1 cent $\$$ every 1 minute of phone calls made by customers, so in this case, the unit volume of traffic is minute. If at one day, the traffic volume was 100 thousand minutes, then the income provider is $=100$ thousand minutes $\times 1$ cent $\$$ per minute $=\$ 1,000$.

3. An optic communications network operator rents out bandwidth to its customers with a tariff of $\$ 5$ per Mbps per month, then in this case, the unit volume of traffic is Mbps. If in a month, operator revenue $=5$ million $\$$, then the traffic volume in a month is $=\$ 5$ million $/ \$ 5$ per Mbps $=1$ million Mbps.

\section{Theoretical Model of Telecommunication Traffic}

Theoretical model of telecommunication traffic can be described as follows:

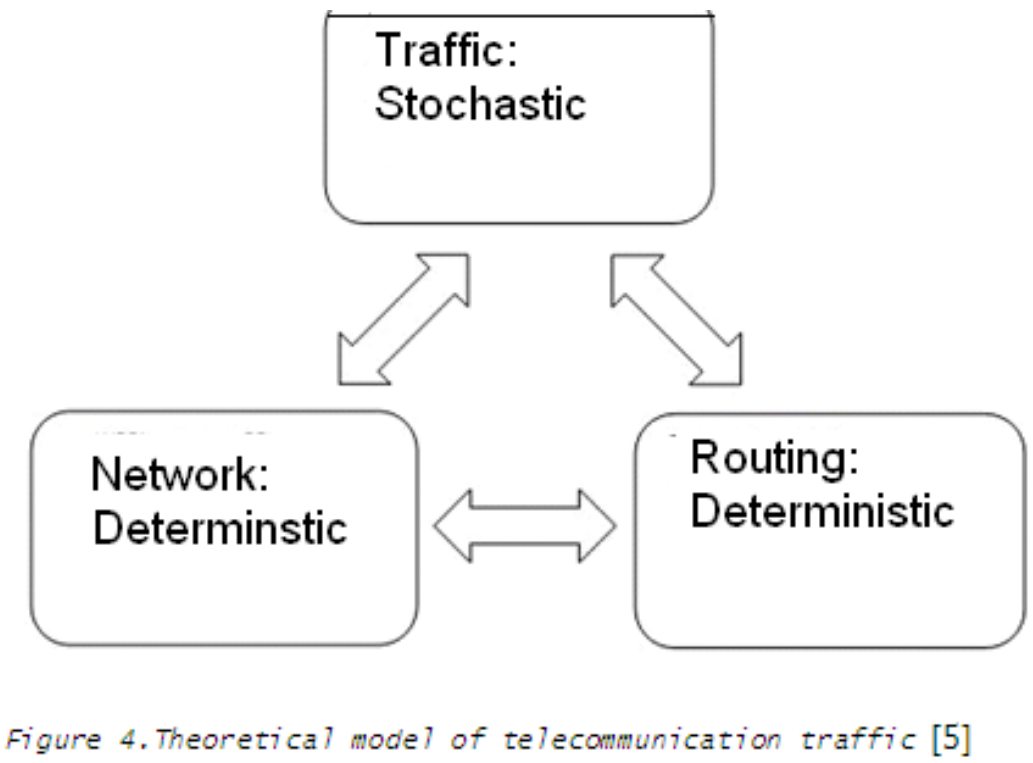


It appears that the theory of telecommunication traffic is a combination of stochastic and deterministic processes. Telecommunication traffic should be expressed in the form of a mathematical model that is most appropriate. In order to select the most appropriate mathematical model, to do the following steps:

\section{i. Observations:}

- Drawing the telecommunications system topology with all its elements, if you already operate a telecommunication system equipped with the capacity of all elements.

\section{ii. Modeling:}

○ Formulation of Mathematical Models Hypothetical collects a variety of mathematical models that are common and usually applied to the telecommunication traffic.

\section{iii. Deduction:}

○ Selecting a mathematical model of the most likely approach the nature and characteristics of traffic on telecommunication system is being designed or are being analyzed

\section{iv. Measurement:}

- Observation and measurement of telecommunication traffic

\section{v. Verification:}

○ Verification of data with a mathematical model

\section{Traffic Process}

Traffic process is presented in Figure 5. The figure illustrates the terminology applied to the traffic. In the IP (Internet Protocol) traffic, the traffic - process describes the process experienced by packets. Because IP-based network is the concept of the present telecommunications system, then in this book, the most frequent form of traffic is discussed as a packet.

It should be noted that there is a difference between the time intervals and instants of time. Inter-arrival time is the length of time between the arrivals of the packet with the next packet. 


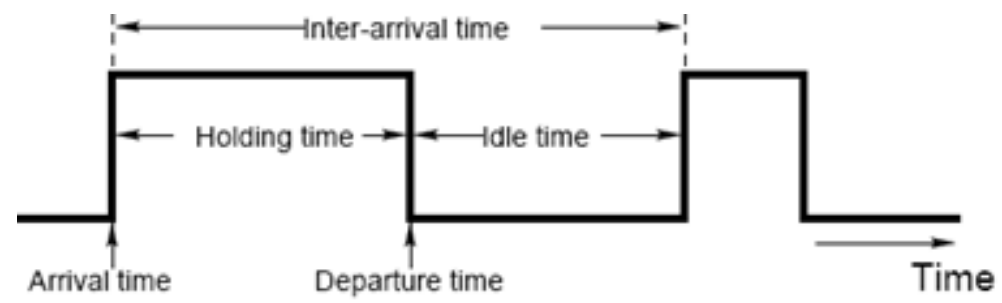

Figure 5. Traffic Process [6]

The application of the concept of point-process of telecommunication traffic engineering is to assume that the arrival of traffic on telecommunication system is a process that can be described at different points on the time scale. For example, there are 1000 packet came in a period $[0, T]$, and then the first packet is assumed to come at the time $t_{1}$, the second pack comes at a time $\mathrm{t}_{2}, \ldots$ hundredth packet comes at $\mathrm{T}_{100}$. With the provision: $0<\mathrm{t}_{1}<\mathrm{t}_{2}<. . \mathrm{t}_{100}$ $<\mathrm{T}$.

It can be concluded that the application of the concept of point-process of telecommunication traffic engineering, traffic is nothing specific observations on how the traffic was coming. So the main principles which are the basis of the theory are the arrival process theory was evaluated using a point process theory.

\section{Traffic at Packet Level}

Based on the level, the most basic type of traffic can be categorized in the packet level. Observations, modelling and measuring traffic at the packet level means observing the traffic that comes to the access network and switching network in its basic form as a packet. To consider here is the variety of things related to the arrival of the packet, regardless of the position of the packet in the group of packets. Observed among others:

\subsection{Packet Size:}

Each packet based on the observed number of bits or bytes of it, including its header. In addition to units of bits or bytes, the packet size can be expressed in units of seconds. For example the size of the packet size $=100$ bits per packet transmitted at speeds of $100 \mathrm{M}$ bits per second, the size of the packet can be expressed with units of seconds $=100$ bits $/(100 \mathrm{M}$ bits per second $)=1$ micro second .

\subsection{Arrival Time Interval:}

Otherwise, regular with time unit, is the interval of time between the arrivals of the packet. As the reference is now coming, not at the end. Suppose $n$ packets to a size 1 millisecond came at ten, more 0 minutes, 0 seconds more, it means the end of the 
packet came in at ten o'clock, more 0 minutes, over 0.001 seconds. Packet number (n +1 ) whose size is 10 milliseconds came at ten o'clock, more 0 minutes, 0.101 seconds and ended more coming at ten o'clock, more 0 minutes, over 0.111 seconds. The interval between the arrivals of these two packets is 0.101 seconds $=101$ milliseconds.

\section{Description of Point Processes}

Packet arrival process described mathematically through a process called stochastic-pointprocesses. The principle of the point-process, is that we can distinguish two successive incoming packets, the packet $\mathrm{i}$-th and packet $(\mathrm{i}+1)$-th always come at different times. The difference in time of arrival of two successive incoming packets called inter-arrival time.

Observation of the arrival of the packet is not included in the point-process events. Information related to one or two packets, the packet command / control, only used to determine whether the arrival of a group of packets of the thousands or millions, believed to have been successfully processed in telecommunication systems or not.

Mathematical theory of the point-process invented and developed by a Swedish mathematician, Connie Palm in about 1940'an. Then the theory is refined by Khintchine in 1968.

Consider Figure 6. The arrival process of the packet in a telecommunications network. Packets that came depicted on each different time scales. Packet i-th comes on $\mathrm{T}_{\mathrm{i}}$ :

$$
0=\mathrm{T}_{0}<\mathrm{T}_{1}<\mathrm{T}_{2}<\ldots<\mathrm{T}_{\mathrm{i}}<\mathrm{T}_{\mathrm{i}+1}<\ldots
$$

The number of packets in the half open interval $[0, t]$ is written with the notation Nt. In this case $\mathrm{Nt}$ is a random variable with continuous time parameter in a discrete space. Nt never shrinks though not enlarged.

The time intervals between two successive incoming packets are:

$$
\mathrm{X}_{\mathrm{i}}=\mathrm{T}_{\mathrm{i}}-\mathrm{T}_{\mathrm{i}-1}, \mathrm{i}=1,2, .
$$

This is called the inter-arrival time, and the distribution is called inter-arrival-timedistribution. 

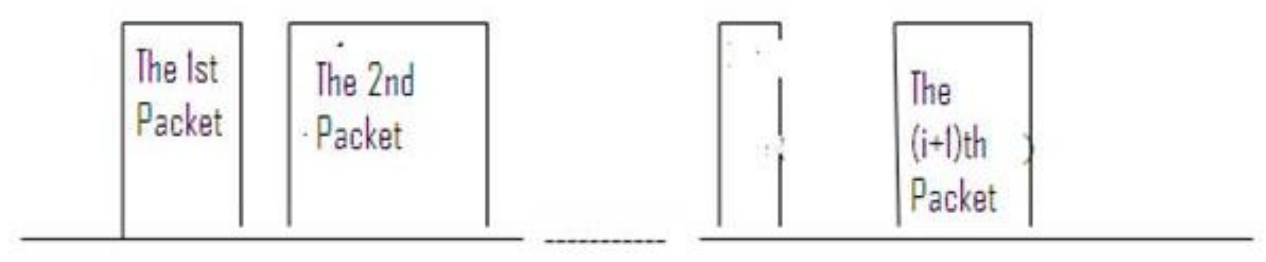
$\begin{array}{ll}T_{0} & T_{1}\end{array}$
$\mathrm{T}_{2}$
$T_{i}$
$\mathrm{T}_{\mathrm{i}+1}$

Figure 6. Point Process Concept [1]

Analysis of point-process from the arrival of packets in telecommunication networks raises two random variables, $\mathrm{Nt}$ and $\mathrm{Xi}$. The meanings of the two random variables are:

\section{i. $\quad$ Number Representation $=\mathbf{N t}$}

This variable will be random if the time interval $\mathrm{t}$ is kept constant and then we observe $\mathrm{Nt}$ for some packets during time $t$.

\section{ii. Interval Representation $=\mathbf{X i}$}

The number of incoming packets is kept constant, then we observe Ti for an interval of time such that the number of incoming packets $=\mathrm{n}$.

\subsection{Fundamental Relationships between $\mathrm{Nt}$ and $\mathrm{Xi}$}

From Figure 6 clear that the number of packets at certain time intervals Nt, will always be smaller than $\mathrm{n}$ if and only if the arrival of the $\mathrm{n}$-th packet equal to the sum of all inter-arrival time is $\geq$ than the entire packet $t$

The above statement can be written as follows:

$$
\text { For } \mathrm{n}=1,2, \ldots \ldots \ldots \text { apply: }
$$$$
N_{t}<n
$$

if and only if:

$$
T_{n}=\sum_{i=1}^{n} X_{i} \geq t
$$

In other words, the above statement can be written as Feller-Jensen's identity as follows:

$$
p\left\{n_{t}<n\right\}=p\left\{T_{n} \geq t\right\}
$$




\subsection{Properties of Number Representation}

There are three basic properties of $\mathrm{Nt}$ :

i. The number of packets that arrive in the interval $[\mathrm{t} 1, \mathrm{t} 2]$ is equal to the NT2 - NT1. The average number of packets that arrive at the same time interval called the renewal function $\mathrm{H}$, where $\mathrm{H}(\mathrm{t} 1, \mathrm{t} 2)=\mathrm{E}\{\mathrm{NT} 2-\mathrm{NT} 1\}$

ii. Density of the packets that arrive during $t$ is:

$$
\lambda_{t}=\lim _{\Delta t \rightarrow 0} \frac{N_{t+\Delta t}-N_{t}}{\Delta t}=N_{t}^{\prime}
$$

iii. lo explain the variation in the arrival process during the time interval $t$ is defined Index of Dispersion for Counts, IDC, which can be written as:

$$
I D C=\frac{\operatorname{Var}\left\{N_{t}\right\}}{E\left\{N_{t}\right)}
$$

The concept of telecommunication traffic measurements corresponding to the representation of the number of packet arrivals is called passive measurement. Measurement devices to records at certain time intervals, the number of packet arrivals. This measurement is usually done with the concept of scanning.. Such measurement is related to the representation of the number of arrivals, which is a fixed time interval

\subsection{Properties of Interval Representation}

There are three basic properties of interval representation, as follows:

1) The distribution $\mathrm{f}(\mathrm{t})$ of the time interval $\mathrm{Xi}$, will result in average packet that comes $\mathrm{m}_{1, \mathrm{i}}=\mathrm{m}_{\mathrm{i}}$. The renewal proccess is a point proccess, but between the two inter-arrival time is a stochastic process that is independent of each other, but both have identical distributions (except X1), called IID (Independent and Identically Distributed)

$$
\begin{gathered}
F_{i}(t)=p\left\{X_{i} \leq t\right\} \\
E\left\{X_{i}\right)=m_{1, i}
\end{gathered}
$$

2) Distribution of the time interval between the beginning of the observation period until the arrival of the first packet written as $V(t)$. The average value of $V(t)$ is the average time measured with a time scale. 
3) To explain the variation in the time interval $t$ is defined Index of Dispersion for Intervals, IDI, which can be written as:

$$
I D I=\frac{\operatorname{Var}\left\{X_{t}\right\}}{E\left\{X_{t}\right)^{2}}
$$

The concept of telecommunication traffic measurements corresponding to the representation of the measurement interval is active. Measurement device records the number of packets in advance for a certain time, and then record the time intervals where there are a number of packets that come with the same amount. Such measurement is related to the representation of the interval. Measurements carried out on a particular service process being actively processed by telecommunications networks.

\subsection{Resume of Point Process}

There are two important things when we study the term point process:

1) Point process raises TWO random variables Nt and Xt. Two random variables have meaning the Number-Representation and Interval-representation.

2) Number-Representation may have different distribution functions than the distribution function of Interval-Representation

\section{Characteristics of Point Process}

There are three basic characteristics of point proccess:

\section{a. Stationary (Homogeneous in Time)}

Practical definition of a stationary nature is, a process is said to be stationary, if the probability distribution describing the processes that are independent of time. Mathematical definition of the nature of the stationary can be written as follows: For $\mathrm{t} 2>0$ and for every $\mathrm{k} \geq 0$, the probability of a packet arrival in the interval $[\mathrm{t} 1, \mathrm{t} 1+\mathrm{t} 2]$ is not dependent on $\mathrm{t} 1$. So for all values of $\mathrm{t}$ and $\mathrm{k}$, we get the equation:

$$
p\left\{N_{t 1+t 2}-N_{t}=k\right\}=p\left\{N_{t 1+t 2+t}-N_{t 1+t}=k\right\}
$$

\section{b. Independent}

This characteristic can be expressed as a change in the current situation where the future of the process depends only on its present state. Mathematical definition: The probability that $\mathrm{k}$ events take place in the interval $[\mathrm{t} 1, \mathrm{t} 1+\mathrm{t} 2]$ is independent of events before time $\mathrm{t} 1$, can be written as: 


$$
p\left\{N_{t 1+t 2}-N_{t}=k \mid N_{t 1}-N_{t 0}=n\right\}=p\left\{N_{t 2}-N_{t 1}=k\right\}
$$

Note: If the above equation holds for all values of $\mathrm{t}$, it is called a Markov process, where changes happen in the future does not depend on how the changes that have been obtained, and commonly referred to as Memoryless. If this property is only valid for a certain point in time, it is called the equilibrium point or points of regeneration. Example in point proccess that occurs at the point of equilibrium is a Poisson process and the measurement process with the concept of scanning

\section{c. Simple}

It has been mentioned before, that at this point we ignore the arrival proccess once some packets at the same time. The process that has these properties is called a simple (simple point process). Examples of the process is not simple is the occurrence of an accident involving several cars at once. Mathematical definition of the simple process is:

$$
p\left\{N_{t+\Delta t}-N_{t} \geq 2\right\}=0 . \Delta t
$$

Here, repeated once again, that the main principles of modelling telecommunications traffic is referring to the point process, where the arrival of the packets described in different time intervals. The difference can be very small, can be enormous. If is a curve that describes the distribution of inter-arrival-time against the time of observation, then obtained a distribution function and density function given appropriate to the situation and condition of traffic and telecommunications networks. This concept, in theory, point process is called interval representation.

Furthermore, also known as the concept of number representation to describe the process that occurs when at some point we observe the number of packets that come in a telecommunications system. If we create a curve that describes the distribution of the number of packets to the time of observation, then obtained a distribution function and density function specified.

Functions of distribution and density that we get through the interval representation are not necessarily the same as we get through the number representation. 
It's important for us to remember. Once again to highlight how an understanding of the concept of point process is very important for us, when I want to explore telecommunication traffic engineering.

Telecommunication traffic engineering experts also present mathematical formulas that can be used practically to perform traffic engineering computations, without having to really know the concept of point process.

For example: Erlang has made a mathematical model to be used in traffic engineering computations in a telephone network, which was then called the formula Erlang-B and Erlang-C formula. Erlang-B formula can be used to carry telephone traffic engineering computations in a network that uses Loss Call Cleared-strategic. In this strategic, that every phone call that failed to get the service would be a loss. While the Erlang-C formula is used to perform traffic engineering computations telephone networks using queuing strategy, that every phone call that failed to get the service will queue first in the buffer and not directly a loss before the buffer is full. Telecommunications network practitioners have been using Erlang formula for nearly 100 years, without the need to understand the true concept of point process. Traffic engineering calculation results proved to be very close to reality traffic measurements.

But when switched networks to IP-based and packet switching, then we all have to explore more about the point process. Many situations and circumstances of the traffic and telecommunications networks which cannot be solved through Erlang formula. The behavior of traffic in an IP-based network must be studied in depth for many years before then compiled a practical formula for the situation and condition of traffic and telecommunications networks.

In this book the theory of probability and stochastic discussed in detail, the reader is invited to read books related references.

All intervals always be positive $(\geq 0)$, and thus can be expressed as a non-negative random variable.. A normal random variable called a variate. The time interval of the process experienced telecommunications traffic, such as a telecommunications service, blocking periods, waiting time, holding, processing time in CPU instructions, inter-arrival time (interarrival times) and others. The distribution function of the time period is a time distribution. In this chapter we will review briefly the theory and statistics that are relevant to the theory of stochastic telecommunication traffic 


\section{Poisson Process}

Poisson process is the most important point proccess. As mentioned on the front, as well as point proccess, characterizing Poisson process is characterized by three things: (a) stationary, (b) Independent and (c) Simple.

The properties of the Poisson process, as well as a point proccess is:

a) Distribution of number-representation is a Poisson

b) Distribution of Interval Representation is Exponential

\section{The concept of the Birth and Death Process}

Already outlined in front of the most important concepts for understanding the behaviour of telecommunication traffic, namely point-process and the arrival-process. The second important concept in telecommunication traffic engineering is a birth and death process which is often used to make the equation of the distribution function of telecommunications traffic.

So again, keep in mind, that the main principles of telecommunication traffic modelling was referring to the point process, where the arrival or completion serves packets depicted at different.

For the practitioner, this concept may make confused. That is why, then the experts then handed a mathematical formula that can be used practically to perform traffic engineering computations, without having to really know the concept of point process. The simplest technique to make the equation of the density function of the telecommunications traffic is by using a birth and death process.

\subsection{Transition Diagrams and Equilibrium Equations}

Consider the transition diagram in Figure 7. That explains the mechanism of birth and death process. 


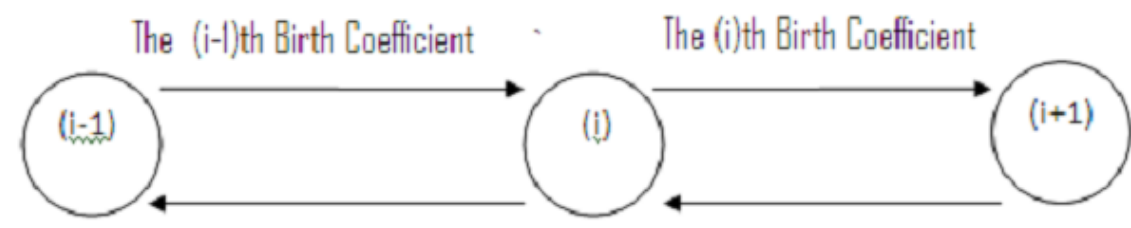

The (i)th Death Coefficient The (i+1)th Death Coefficient

Figure 7. State Transition Diagram of Birth and Death Process

The number (i-1), (i) and (i +1$)$ represents the circumstances when there is a (i-1), or (i) or (i +1 ) packet being served by the server.

The existence of the arrival of a new packet, analogous to a birth rate of coefficients of birth. The existence of a packet or a service that had been served analogous to a death rate of coefficients of birth.

Birth and death process on telecommunication traffic, is the process of increasing the packet comes to asking to be served that are analogous to the birth, while the complete server serves a packet by analogy with death.

Assuming statistical equilibrium happens, and then by using the principle of global balance, applies two equations:

a. Node equations:

The situation that occurred at the beginning of statistical equilibrium, where we only pay attention to state 0 , which means there is no incoming packets. So there are only two possibilities occur:

State 0 will transition to state 1 with a probability of the probability of state $0\{=p$ (0)\} multiplied by the coefficient birth (0), $b(0)$, and

State 1 will transition to state 0 with a probability of the probability of occurrence of state $1\{=p(1)\}$ multiplied by the death coefficient (1), $d(1)$.

Assuming statistical equilibrium has occurred then the two possibilities must be the same, so that it can be written in the form of equation:

$$
p(0) \cdot b(0)=p(1) \cdot d(1)
$$




\section{b. Cut Equations:}

Observations on a random node, the state (i)th.

In the event of statistical equilibrium in state (i), there are four possibilities that occur:

1) State (i) th will transition into state $(i+1)$ th with a probability of the probability of occurrence of state $i\{=p(i)\}$ multiplied by the birth coefficient (i) th $\}, b$ (i)

2) State (i) th will transition into state (i-1)) th with a probability of the probability of occurrence of state $\mathrm{i}\{=\mathrm{p}(\mathrm{i})\}$ multiplied = death coefficient (i) th, d (i)

3) State (i-1) th will transition into state (i) th with a probability of the probability of state (i-1) th $\{=\mathrm{p}(\mathrm{i}-1)\}$ multiplied birth coefficient (i-1), b (i -1)

4) State $(i+1)$ th will transition into state (i)) th with a probability of the probability of state $(i+1)=\{p(i+1)\}$ multiplied by the death coefficient $(i+1)\}, d(i+1)$.

Assuming statistical equilibrium then the number 2 pieces probabilities showing the transition FROM state (i) must be equal to the number 2 pieces probabilities indicating a transition TO the state (i) that can be written one in the form of equations:

$$
p(i) \cdot b(i)+p(i) \cdot d(i)=p(i+1) \cdot d(i+1)+p(i-1) \cdot b(i-1)
$$

Statistical equilibrium equations is the interpretation of the arrival theorem

\section{Creating Mathematical Model of Formula Erlang B}

One implementation of the Birth and Death Process in the field of telecommunication traffic engineering is the Erlang-B formula.

Erlang-B formula is very famous in the telephony-switching circuit. For nearly a hundred years, Erlang B formula was used to calculate the traffic engineering in telecommunications networks. The results of calculations using the Erlang-B formula proved very accurate when compared with real measurement results on a telephone network that is LCC (loss-callcleared).

There are two principles of the use of Birth and Death Process as a model of traffic in telecommunication networks, are summarized in the following:

1) The first is to determine the assumptions of the traffic coming. Erlang-B formula used in the telephone network telephone traffic is referring to the corresponding point process. The rate of arrival of call is $\lambda$ and the service rate is $\mu$. Traffic coming in and 
served on the telecommunications system is assumed to be a PCT-1 (Pure Chance Traffic Type 1). At traffic PCT-1 may be justified to use the average value as the basis, or known as PASTA (Poisson Arrival See Time Arrival). Traffic that comes can be expressed by erlang and written with the notation $\mathrm{A}=\lambda / \mu$

2) The second is that we should be able to describe the transition of the state diagram. For that we need to know how many and how large state-coefficient birth and deathcoefficient in each state.

\subsection{Determination of the number of state}

Telephone network based on circuit-switching, the number of states is equal to the number of channels in the telephone network. This is understandable, since the point of our attention on the network is the probability of a channel being hold. State 0 represents no premium is being hold, state 1 state there is one channel that hold and so on. Easily understandable, that in this situation, the maximum number of states is equal to $\mathrm{n}$, that is equal to the number of channels in telecommunication networks which we observe. So $\mathrm{n}$ is limited.

\subsection{Determination of birth-coefficients and detach-coefficient}

In order to determine the birth-coefficient and death-coefficients, we must understand the mechanisms that occur when handling traffic in the network. If we really understood the mechanism that happens, then we can decide the coefficients. It is important to remember, that we now depend on assumptions. Two assumptions to make the Erlang-B formula are:

a) The first assumption: telephone call came refers to the concept of point process with the arrival rate of call is equal to $\lambda$. Thus the coefficients of the birth of the state 0 into state 1 are equal to $\lambda$.

b) The second assumption: for the telephone call is being hold in the telecommunications channel, will end with a service rate of $\mu$. Thus the death-coefficient of state (1) into state (0) is equal to $\mu$.

c) The third assumption: the number of subscribers is very much in comparison to the number of channels in the network. Thus, the coefficient of births in the network circuit switching loss is always equal to $\lambda$. Why is that? Put simply be understood as follows. Imagine a situation when no call came, there were a very large number of customers, say mathematically regarded as being one of an infinite potential to make a call, at a rate 
equal to $\lambda$, this is the birth-coefficient from state 0 into state 1 . Then we imagine when it was first call on the network is still being served. There are a number of customers who are very much reduced by 1 , i.e., who is doing call and cause the state to state 1 , of course, the number of subscribers is still a lot, because mathematically: infinity minus one will be equal to infinity. Thus the potential for a second customer will do call would have probability $=\lambda$. So the coefficient of birth state 1 into state 2 is also equal to $\lambda$. As the number of subscribers is assumed remarkable than the number of channels, it is understood that all birth-coefficient would be equal to $\lambda$.

d) In contrast to the determination of the coefficient of birth, which we consider are the potential customers who do not call, because the call is analogous to the increase in the birth. When determining death-coefficient, which we noticed was the call is holding. The existence of call that has been served we analogy with death. The number of call Maximum $=\mathrm{n}$. Then the number of deaths is also limited. Easily, we can understand, if the coefficient death of a call equal to $\mu$, then the potential is there any choice of 2 call to be completed serviced is $2 \mu$. Mean death-coefficient of the state 2 is equal to $2 \mu$, etc. Similarly, the coefficient of the death state $i$ is equal to i. $\mu$.

The model of the Loss-Call-Cleared based phone network presented in Figure 8.

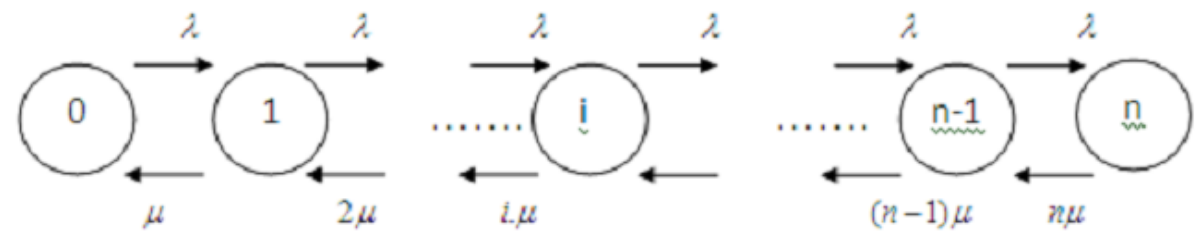

Figure 8. Formula Er7ang-B State Transition Diagram

Equilibrium equations of the State Transition Diagram in Figure 8. Written as follows;

State 0: $\lambda \lambda \cdot p(0)=\mu \cdot p(1 \rightarrow \lambda \cdot p(0)=\mu \cdot p(1) \rightarrow A \cdot p(0)=p(1)$

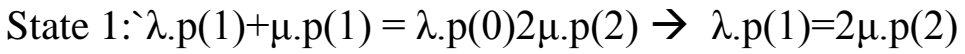

State $2:^{\prime} \lambda \cdot \mathrm{p}(2)+2 \mu \cdot \mathrm{p}(2)=\lambda \cdot \mathrm{p}(1)+3 \mu \cdot \mathrm{p}(3) \rightarrow \lambda \cdot \mathrm{p}(2)=3 \mu \cdot \mathrm{p}(3)$ 
State (i-1): $\quad \lambda \cdot p(i-1)=(i) \mu \cdot p(i)$

State (i): $\quad \lambda \cdot p(i)=(i+1) \mu \cdot p(i+1)$

State $(\mathrm{i}+1): \quad \lambda \cdot \mathrm{p}(\mathrm{i}+1)=(\mathrm{i}+2) \mu \cdot \mathrm{p}(\mathrm{i}+2)$

In telephone networks - circuit switched - loss call cleared: $\lambda / \mu=$ offered traffic $=\mathrm{A}$

Then the equilibrium equations can be written into:

$$
\begin{aligned}
& \mathrm{p}(0)=\mathrm{p}(0) \\
& \mathrm{p}(1)=\mathrm{A} \cdot \mathrm{p}(0) \\
& p(2)=\frac{A}{2} p(1)=\frac{A^{2}}{2} p(0)
\end{aligned}
$$

$$
p(n)=\frac{A}{(n)} p(n-1) \quad=\frac{A^{n}}{(n) !} p(0)
$$

We can compute $p$ (i) only as a function of A and i only, by way of substitution $p(0)$ into the equation involving only A and i only. For that, we must recall the principles of probability theory in this situation: the sum of all probabilities $=1 \rightarrow p(0)+p(1)+p(2)+$ $\mathrm{P}(\mathrm{n})=1$.

$$
\begin{array}{r}
p(o)+p(1)+p(2)+\ldots \ldots . . p(n)=1 \\
p(o)+\frac{A}{1} p(0)+\frac{A^{2}}{2 !} p(0)+\ldots \ldots . . \frac{A^{n}}{n !} p(0)=1 \\
p(o)=\frac{1}{1+A+\frac{A^{2}}{2 !}+\ldots \ldots \ldots . . . \frac{A^{n}}{n !}}
\end{array}
$$

Then it can be derived loss system, formula or already known as the Erlang-B Formula: 


$$
\operatorname{En}(A)=p(n)=\frac{\frac{A^{n}}{n !}}{1+A+\frac{A^{2}}{2 !}+\ldots \ldots \ldots . . \frac{A^{n}}{n !}}
$$

For practical calculations that do not require high accuracy, then we can use erlang-table, as provided in the annex. By knowing two variables we can determine the unknown third variable. Suppose we know the traffic offered (A) and the number of channels, then we can know how much En (A) or commonly referred to as the probability of loss, and are often written with the notation B or Pn (A).

\subsection{Recursive Formula Erlang-B}

Calculations involving three elements: A, $n$ and En (A) turns out using the formula is quite complicated to be solved using a calculator. For it is a recursive formula for making computer program.

$$
E_{x}(A)=\frac{A \cdot E_{x-1}(A)}{x+A \cdot E_{x-1}(A)} ; \quad E_{0}(A)=1
$$

\subsection{Erlang-B Formula Problem \& Solution}

A trunk-network consists of 10 channels serve traffic with the following characteristics: (a) Structure-channel group is homogeneous and full accesibility (b) traffic served by LCC = Lost Call Cleared (c) Arrival traffic is a Poisson arrival process with rate arrival $=\lambda=500$ call / sec, and (d) Service-time distributed eksonential-intensity $=\mu=100 \mathrm{sec} / \mathrm{call}$.

Calculate:

(a) Time congestion, call congestion and traffic congestion

(b) Offered traffic, traffic \& Carried traffic loss (c) Provider income on a day when the average arrival rate for 23 hours non-busy hour arrival rate $=0.75$ during rush hour, and if any call on average provide $0.05 \$$

(d) The average utilization of

SOLUtion

$\mathrm{A}=\lambda / \mu=500 / 100=5 ; \mathrm{n}=10$ 
(a) $\mathrm{E}_{10}(5)=0,0184$

Time congestion, call congestion \& traffic congestion $=\mathrm{E}=\mathrm{B}=\mathrm{C}=0,0184$

(b)Offered traffic $=\mathrm{A}=5$ erlang

Loss traffic $=$ traffic congestion $*$ Offered traffic $=\mathrm{R}=\mathrm{A}_{1}=5 * 0,0184=0,0919$ erlang

Carried Traffic $=$ Ac $=\mathrm{Y}=\mathrm{A}-\mathrm{R}=5-0,0919=4,9081$ erlang

(c)Call congestion $=\mathrm{B}=0,0184$ and $\lambda=500 \mathrm{call} / \mathrm{sec} \rightarrow$ number of carried call in 1 busy hour $=500 *(1-\mathrm{B}) * 3600=1,766,908$

Number of call carried in a day $=$ call in busy hour +23 hoyr non-busy hour $=1766908+$ $(23 * 0,75 * 1,766,908)=32,246,067$ call

Provider income in a day $=32,246,067$ call $* 0.05 \$ /$ call $=1,612,303.35 \$$

(d)Average Utilisation in erlang/channel=

$$
a_{i}=a=\frac{Y}{n}=\frac{A\left\{1-E_{n}(A)\right\}}{n}=4.9 / 10=0.49
$$

\section{Creating Mathematical Model of Formula Erlang A or First Formula Erlang}

Erlang formulas are well known use in telecommunications networks, especially for the use of direct telephony service. Erlang-B is used for loss-system and Erlang-C for queuingsystem. Many have asked whether there is never a formula Erlang-A?

The answer, in fact there, but because it is rarely used in practical implementation, the Erlang formula-A or the so-called Erlang's first formula to less well known.

Erlang is a mathematician who first popularized the application of mathematical equations in the modelling of telephone networks. It has been over 100 years before the existing other mathematicians, Poisson, who has applied the concept of point process called Poisson process. Erlang then adapted so that better suit the practical measurement of telephone traffic.

The derive of Poisson distribution formula, which was adapted by Erlang is similar to the decrease in Erlang-B formula, but used to state an infinite number. Thus, the determination of the probability of state 0 is: 


$$
\begin{gathered}
p(o)+p(1)+p(2)+\ldots \ldots . . p(i)=1 ; i \rightarrow \infty \\
p(o)+\frac{A}{1} p(0)+\frac{A^{2}}{2 !} p(0)+\ldots \ldots . . \frac{A^{i}}{i !} p(0)=1 ; i \rightarrow \infty \\
p(o)=\frac{1}{e^{A}}
\end{gathered}
$$

Then the Poisson distribution derived using the birth and death process:

$$
p(i)=\frac{A^{i}}{i !} \cdot e^{-A}
$$

When first derived formula, Erlang adapting Poisson distribution, assuming that the number of telephone lines is limited, the amount of state in the state transition diagram would also limited.

Then $\mathrm{p}(0)$ is :

$$
p(o)=\left\{\sum_{j=0}^{n} \frac{A^{j}}{j !}\right\}^{-1}
$$

And the formula Erlang-A, or Erlang-first, or a truncated poisson distribution:

$$
p(i)=\frac{\frac{A^{i}}{i !}}{\sum_{j=0}^{n} \frac{A^{j}}{j !}}
$$

\section{Creating Mathematical Model of Formula Erlang $\mathbf{C}$}

Erlang-A Formula (also known as the first erlang formula) and Erlang-B formula known use in loss-network have been described before. Erlang is not just having been analyzed lossnetwork, but also have been analyzed queuing networks. Although many new queuing networks use today, but relevant formulas for queuing network has been derived by experts from 100 year ago. Formula developed by Erlang for queuing-network called the Erlang-C formula. At a later date, since D.G.Kendall promote notation for the network queue, then the formula erlang-C is suitable for the use of the network queue $M / M / n$. 
The formula consists of three variables, in contrast to the original standard, indicating that the variable $S$ (number of users) is very much and formulas can be applied generally to all queuing disciplines.

State transition diagram of the network queue M / M / n are presented in Figure 9.

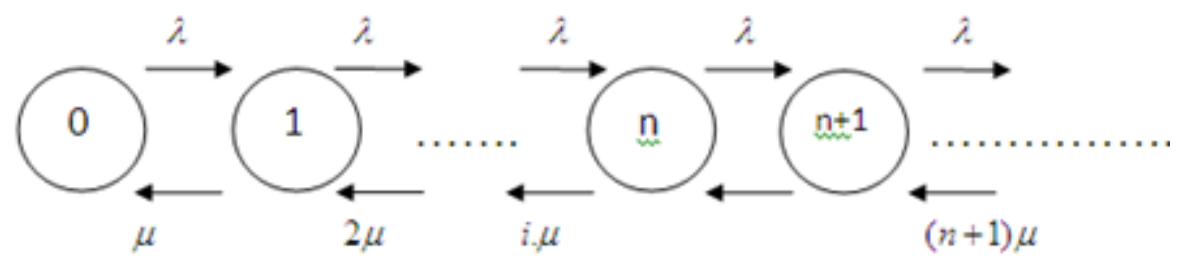

Figure 9. State transition diagram of the network queue $\mathrm{M} / \mathrm{M} / \mathrm{n}$

The difference between the queuing-network compared to the loss-network is the buffer innetwork queuing, this causes the state transition diagram of the state are queuing-network (n +1 ) and so on. Thus the deriving of formula for the network queue M / M / $\mathrm{n}$ as follows:

Following the concept of global equilibrium equations balance:

$$
\begin{aligned}
& \lambda \cdot p(0)=\mu \cdot p(1) \\
& \lambda \cdot p(1)=2 \mu \cdot p(2)
\end{aligned}
$$

$$
\lambda \cdot p(i)=(i+1) \cdot \mu \cdot p(i+1)
$$

$$
\begin{aligned}
& \lambda \cdot p(n-1)=n \cdot \mu \cdot p(n) \\
& \lambda \cdot p(n)=n \cdot \mu \cdot p(n+1)
\end{aligned}
$$




$$
\lambda \cdot p(n+j)=n \cdot \mu \cdot p(n+j+1)
$$

Thus, the hallmark of the network equilibrium equations in the queue is the death-coefficient on state $\mathrm{p}(\mathrm{n}-1), \mathrm{p}(\mathrm{n})$ $(\mathrm{pn}+\mathrm{j})$ is always equal to the state probability multiplied by n. $\mu$

Since $A=\lambda / \mu$ then:

$$
\begin{gathered}
p(i)=\frac{A^{i}}{i !} p(0) \quad \text { for }: 0 \leq i \leq n \\
p(i)=\left[\frac{A}{n}\right]^{i-n} \cdot p(0)=\frac{A^{i}}{n ! n^{i-n}} p(n) \quad i \geq n
\end{gathered}
$$

Since total probability of all state $=1$

$$
\begin{aligned}
& p(0)+p(1)+\ldots \ldots . . p(n) \ldots \ldots \ldots \ldots \ldots \ldots \ldots \ldots . . .=1 \\
& p(0)\left\{1+\frac{A}{1}+\frac{A^{2}}{2 !}+\ldots \ldots \ldots \ldots \frac{A^{n}}{n !}+\ldots \ldots \ldots \ldots . . .\right\}=1 \\
& p(0)\left\{1+\frac{A}{1}+\frac{A^{2}}{2 !}+\ldots \ldots \ldots . . \frac{A^{n}}{n !}\left(1+\frac{A}{1}+\frac{A^{2}}{2 !}+\ldots \ldots \ldots\right)\right\}=1
\end{aligned}
$$

Applies to all telecommunications networks, the average value of traffic should be less than the capacity or the number of servers, then the equilibrium will only happen if $\mathrm{A}<\mathrm{n}$.

Then:

$$
p(0)=\frac{1}{\sum_{i=0}^{n-1}\left[\frac{A^{i}}{i !}+\frac{A^{n}}{n !} \cdot \frac{n}{n-A}\right]}
$$

Erlang-C formula used to calculate the probability of a packet or a job will be waiting to be written as follows: 


$$
\begin{gathered}
E_{2, n}(A)=p\{W>0)=p(n) \cdot \frac{n}{n-A} \\
E_{2, n}(A)=p\{W>0)=\frac{\frac{A^{n}}{n !} \cdot \frac{n}{n-A}}{1+\frac{A}{1}+\frac{A^{2}}{2 !}+\ldots \ldots \cdot \frac{A^{n-1}}{(n-1) !}+\frac{A^{n}}{n !} \cdot \frac{n}{n-A}} \quad \text { (27) for }:: A<n
\end{gathered}
$$

There are two formulas to calculate the probability of queuing on the network queue M / M / $\mathrm{n}\{\mathrm{E} 2, \mathrm{n}(\mathrm{A})\}$ by using the Erlang B formula $\{\mathrm{E} 1, \mathrm{n}(\mathrm{A})\}$ as follows:

$$
\begin{aligned}
& E_{2, n}(A)= p\{W>0)=\frac{n \cdot E_{1, n}(A)}{n-A\left\{1-E_{1, n}(A)\right\}} \\
& \frac{1}{E_{2, n}(A)}=\frac{1}{E_{1, n}(A)}-\frac{1}{E_{1, n-1}(A)}
\end{aligned}
$$

The notation for the probability of queue, in addition to $E 2, n(A)$ and $p\{w>0)$ is also commonly written as D or D (A).

We can also calculate the average number of queues that are in the buffer $=$ Ln (a unit $=$ packet or request) and the average number of queuing $=\mathrm{Lnq}$ (with units $=$ packet or requests per second).

$$
L_{n}=E_{2, n}(A) \cdot \frac{A}{n-A}
$$

$$
L_{n q}=\frac{L_{n}}{p\{L>0\}}=\frac{n}{n-A}
$$

By writing $\mathrm{s}=$ average service time $=1 / \mu$,

the average time to queue for the queue packets only (w) and the average queuing time of the entire packet $(\mathrm{W})$ is calculated by the following formula:

$$
\begin{gathered}
W_{n}=E_{2, n}(A) \cdot \frac{s}{n-A} \\
w_{n}=\frac{s}{n-A}
\end{gathered}
$$


The average queuing time for all packets often referred to as the response time. The calculation of the average queuing time of the above can be used for queuing systems that use only one server that is by replacing $\mathrm{n}$ with the number 1 .

\section{Biography}

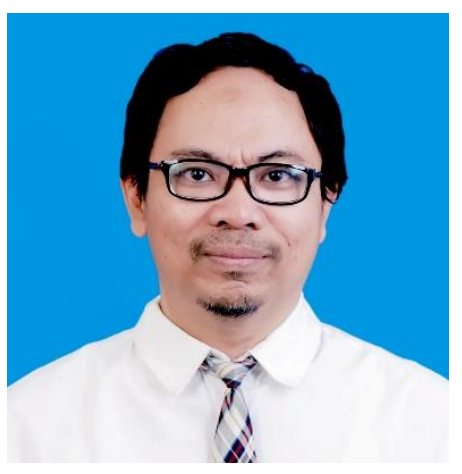

Sigit Haryadi is lecturer in ITB (Bandung Institute of Technology), Indonesia, since 1984.

\section{References}

[1] ITU-D. (2005). Teletraffic Engineering Handbook. ITU-D Study Group 2 Question 16/2. $328 \mathrm{pp}$.

[2] Sigit Haryadi. (1984). Teletraffic Engineering Lecture Notes \& Handout. Institut Teknologi Bandung. 1984 - 2018.

[3] Rabe, F.W. (1949). Variations of telephone traffic. Electrical Communications, Vol. 26 (1949) 243-248.

[4] Karlsson, S.A. (1937). Tekniska anordninger f"or samtalsdebitering enligt tid (Technical arrangement for charging calls according to time, In Swedish). Helsingfors Telefonforening, Tekniska Meddelanden 1937, No. 2, pp. 32-48.

[5] ITU-D. (2005). Teletraffic Engineering Hansbook. ITU-D Study Group 2 Question $16 / 2$. Chapter 1. pp.2

[6] ITU-D. (2005). Teletraffic Engineering Hansbook. ITU-D Study Group 2 Question 16/2. Chapter 1. pp.4

[7] Palm, C. (1941). M“attnoggrannhet vid best"amning af trafikm“angd enligt genomsokningsforfarandet (Accuracy of measurements in determining traffic volumes by the scanning method). Tekn. Medd. K. Telegr. Styr., 1941, No. 7-9, pp. 97-115

[8] Khintchine, A.Y. (1955). Mathematical methods in the theory of queueing. London 1960. 124 pp. (Original in Russian, 1955)

[9] Engset, T.O. (1918). Die Wahrscheinlichkeitsrechnung zur Bestimmung der W“ahlerzahl in automatischen Fernsprech"amtern. Elektrotechnische Zeitschrift, 1918, Heft 31. Translated to English in Telektronikk (Norwegian), June 1991, 4pp.

[10] Kruithof, J. (1937). Telefoonverkehrsrekening. De Ingenieur, Vol. 52 (1937) : E15-E25

[11] Kendall, D.G. (1951). Some problems in the theory of queues. Journal of Royal Statistical Society, Series B, Vol. 13 (1951) : 2, 151-173. 
[12] Little, J.D.C. (1961). A proof for the queueing formula $\mathrm{L}=$ W. Operations Research, Vol. 9 (1961) : 383-387.

[13] Khintchine, A.Y. (1955). Mathematical methods in the theory of queueing. London 1960. 124 pp. (Original in Russian, 1955).

[14] Jackson, J.R. (1963). Jobshop-like queueing systems. Management Science, Vol. 10 (1963), No. 1, pp. 131-142

[15] Kleinrock, L. (1975). Queueing systems. Vol. I: Theory. New York 1975. 417 $\mathrm{pp}$

[16] Kleinrock, L. (1976). Queueing systems. Vol. II: Computer applications. New York 1976. 549 pp.

[17] Conway, A.E. \& Georganas, N.D. (1989). Queueing networks - exact computational algorithms: A unified theory based on decomposition and aggregation. The MIT Press 1989. 234 pp

[18] Jensen, Arne (1950). Moe's Principle - An econometric investigation intended as an aid in dimensioning and managing telephone plant. Theory and Tables.

Copenhagen 1950. $165 \mathrm{pp}$.

[19] Riordan, J. (1956). Derivation of moments of overflow traffic. Appendix 1 (pp. 507-514) in (Wilkinson, 1956

[20] Rapp, Y. (1965). Planning of junction network in a multi-exchange area. Ericsson Technics 1965, No. 2, pp. 187-240

[21] Wilkinson, R.I. (1956). Theories for toll traffic engineering in the U.S.A. The Bell System Technical Journal, Vol. 35 (1956) 421-514.

[22] Hayward, W.S. Jr. (1952). The reliability of telephone traffic load measurements by switch counts. The Bell System Technical Journal, Vol. 31 (1952) : 2, 357-377

[23] Fredericks, A.A. (1980). Congestion in blocking systems - a simple approximation technique. The Bell System Technical Journal, Vol. 59 (1980) : 6, 805-827

[24] Andre Febrian Kasmar; Sigit Haryadi. (July 2017). Delay analysis on load balancing NDN, case study: Palapa ring. The 3rd International Conference on Wireless and Telematics (ICWT), in Indonesia.

[25] Ardi Syawaldipa; Sigit Haryadi. (July 2017). Packet drop analysis on NDN's content distribution, case study: Palapa ring topology. The 3rd International Conference on Wireless and Telematics (ICWT), in Indonesia.

[26] Astari Nandhiasa; Sigit Haryadi. (2015). Indonesian regulation management recommendation for Over-the-Top services. 1st International Conference on Wireless and Telematics (ICWT), at Manado, Indonesia.

[27] Baik Budi; Sigit Haryadi. (2016). Simulasi Mobility pada Software Defined Networking. Seminar Nasional Teknik Elektro UIN Sunan Gunung Djati 2016, at Bandung, Indonesia.

[28] Bambang Supriadi; Sigit Haryadi. (2016). An academic study of roadmap of $5 \mathrm{G}$ implementation in Indonesia. The 10th International Conference on Telecommunication Systems, Services, and Applications (TSSA), At Denpasar, Indonesia.

[29] Dwina Fitriyandini Siswanto; Sigit Haryadi. (2016). Broadband user demand forecasting in Indonesia based on Fourier analysis. 10th International Conference on Telecommunication Systems Services and Applications (TSSA), Indonesia.

[30] Dyah Rakhma Ariyanti; Sigit Haryadi. (October 2017). Analysis of Harmony In Gradation Index on 5G Cellular Network Quantitative Analysis. The 11th 
International Conference on Telecommunication Systems, Services, and Applications, At Lombok, Indonesia.

[31] Elsa Vinietta; Sigit Haryadi. (2015). Hubber strategy and regulation. 1st International Conference on Wireless and Telematics (ICWT), at Manado, Indonesia.

[32] Fidyatun Nisa; Sigit Haryadi. (2016). Simulation of the fault management with Self Healing mechanism (case study: LTE Network in Banda Aceh Area). 10th International Conference on Telecommunication Systems Services and Applications (TSSA), Indonesia.

[33] Harashta Tatimma Larasati; Sigit Haryadi. (2016). Basic planning of data center infrastructure and bandwidth requirement in integrated government network. 2nd International Conference on Wireless and Telematics (ICWT), in Yogyakarta, Indonesia.

[34] Haryadi, S. (2017). Internet Services Pricing Regulation. Researchgate. DOI10.13140/RG.2.2.28217.16487.

[35] Haryadi, S. (2018, February 26). Measurement of Cellular Network's Availability and Reliability based on the Signaling Ladder Diagram. Retrieved from osf.io/preprints/inarxiv/hwvm8

[36] Haryadi, S. (2018, February 27). Performance Measurement of Telephone Network and Service. Retrieved from osf.io/preprints/inarxiv/5c7qa

[37] Haryadi, S. (2018, January 25). Chapter 1. The Concept of Telecommunication Network Performance and Quality of Service. Retrieved from osf.io/mukqb

[38] Haryadi, S. (2018, January 26). Chapter 2 of Network Performance and Quality of Service: Determination of Key Performance Indicator (KPI). Retrieved from osf.io/preprints/inarxiv/6gtnd

[39] Haryadi, S. (2018, January 26). Chapter 3 of Network Performance and Quality of Service: Technical Measurement of a Mobile Network Performance and Quality of Service. Retrieved from osf.io/q4wsz

[40] Haryadi, S. (2018, January 30). Basic Calculation of the Network's Availability and Reliability (Chapter 4 of Network Performance and Quality of Service). Retrieved from osf.io/preprints/inarxiv/z5mwq

[41] Haryadi, S. (2018, March 4). Performance Measurement of SMS and/or Chat Service. Retrieved from osf.io/preprints/inarxiv/h76uc

[42] Haryadi, Sigit; Andina, Raisha. (2012). QoS Measurement of File Transfer Protocol Services In 3 G Networks Using Aggregation Method. Denpasar, Indonesia. 2012.

[43] Haryadi, Sigit; Limampauw, Ivantius. (2012). QoS Measurement of Telephony Services In 3G Networks Using Aggregation Method. Conference Proceeding of TSSA 2012. Denpasar, Indonesia.

[44] Haryadi, Sigit; Nusantara, Sandy. (2012). QoS Measurement of Web Browsing Services In 3G Networks Using Aggregation Method. Conference Proceeding of TSSA 2012. Denpasar, Indonesia.

[45] Haryadi, Sigit; Pramudita, Arnold. (2012). QoS Measurement of Video Streaming Services in a 3G Networks Using Aggregation Method. Conference Proceeding of TSSA 2012. Denpasar, Indonesia.

[46] Maya Rahayu; Sigit Haryadi; Dyah Rakhma Aryanti. (2015). Over the Top chat service key performance indicator. 1st International Conference on Wireless and Telematics (ICWT), Indonesia.

[47] Muhammad Najib Dwi Satria; Sigit Haryadi. (October 2017). Effect of the content store size to the performance of named data networking: Case study on Palapa 
Ring topology. The 11th International Conference on Telecommunication Systems, Services, and Applications, At Lombok, Indonesia.

[48] Muhammad Rizky Hikmatullah; Sigit Haryadi. (2017). Perceptual evaluation of speech quality over the top call service. 3rd International Conference on Wireless and Telematics (ICWT), Indonesia.

[49] Rizna Nofitasari; Sigit Haryadi. (2017). Self-healing mechanism with RSRP measurement in LTE network. 3rd International Conference on Wireless and Telematics (ICWT), Indonesia.

[50] Sigit Haryadi. (2005). Mobile Switching Centre Processing Capacity Computations. WPMC. Alborg, Sweden.

[51] Sigit Haryadi. (2013). Telecommunication Service and Experience Quality. Lantip Safari Media, Bandung, Indonesia. ISBN: 978-602-18578-6-1.

[52] Sigit Haryadi. (2013). Telecommunication Traffic: Technical and Business Consideration. Lantip Safari Media, Bandung, Indonesia. ISBN: 978-602-18578-5-4.

[53] Sigit Haryadi. (2016). Grade of Service \& Quality of Service Concept and Analysis. Researchgate. Available online at https://www.researchgate.net/publication/313101070_Grade_of_Service_Quality_of_Service Concept and Analysis

[54] Sigit Haryadi. (2016). Performance Indicator of Telecommunication Service. Researchgate. Available online at https://www.researchgate.net/publication/313101244 Performance Indicator of Telecommu nication_Service.

[55] Sigit Haryadi. (2016). Telecommunication Quality of Service Concept. Research gate. Available online at https://www.researchgate.net/publication/313101013_Telecommunication_Quality_of_Servic e_Concept

[56] Sigit Haryadi. (2016). Telecommunication Traffic Unit and Traffic Mathematical Model. Researchgate. Available online at https://www.researchgate.net/publication/313101068_Telecommunication_Traffic_Unit_and_ Traffic Mathematical Model

[57] Sigit Haryadi; Angelia Hermawan. (2014). Recommendations for handling price war between telecom operators in Indonesia. The 8th International Conference on Telecommunication Systems, Services, and Applications (TSSA), At Denpasar, Indonesia.

[58] Sigit Haryadi; Dyah Rakhma Ariyanti. (October 2017). The Fairness of Resource Allocation and Its Impact on 5G Ultra-Dense Cellular Network Performance. The 11th International Conference on Telecommunication Systems, Services, and Applications, At Lombok, Indonesia.

[59] Sigit Haryadi; Febrianty. (2015). Recommendation on domestic Internet interconnection towards all-IP network. The 9th International Conference on Telecommunication Systems, Services, and Applications (TSSA), At Denpasar, Indonesia.

[60] Sigit Haryadi; FestyLalita Niramaya. (2014). Study of unfair competition between regulated and unregulated VoIP providers in the mixed of non and all-IP network era. The 8th International Conference on Telecommunication Systems, Services, and Applications (TSSA), At Denpasar, Indonesia.

[61] Sigit Haryadi; Fine Nur Islami. (2014). Analysis of core-stateless fair queuing for fair bandwidth allocation in an IP network. The 8th International Conference on Telecommunication Systems, Services, and Applications (TSSA), At Denpasar, Indonesia. 
[62] Sigit Haryadi; Jalu Ahmad Dirgantoro. (2015). Design of Indonesian hubber communication network. 1st International Conference on Wireless and Telematics (ICWT), at Manado, Indonesia.

[63] Sigit Haryadi; Marisa Premittasari. (2014). Traffic analysis of number of request per user and Volume per Request Hits on IP networks. The 8th International Conference on Telecommunication Systems, Services, and Applications (TSSA), At Denpasar, Indonesia.

[64] Suci Ramadona; Sigit Haryadi; Dyah Rakhma Aryanti. (2015). Over the top call service key performance indicator. 1st International Conference on Wireless and Telematics (ICWT), Indonesia. 